\title{
Fórmulas DRIS para o diagnóstico nutricional de pomares de cupuaçueiros
}

\author{
Paulo Guilherme Salvador Wadt $\left({ }^{1 *}\right)$; Jairo Rafael Machado Dias (2); Daniel Vidal Perez (3); \\ Cleigiane de Oliveira Lemos (') \\ (') Embrapa Acre, Caixa Postal 321, 69908-970 Rio Branco (AC). \\ (2) Universidade Federal do Acre, Programa de Pós-graduação em Produção Vegetal, Caixa Postal 500, 69915-900 Rio Branco (AC). \\ (3) Embrapa Solos, Caixa Postal 1024, 22426-000 Rio de Janeiro (RJ). \\ (*) Autor correspondente: paulo@cpafac.embrapa.br
}

Recebido:13/mai./2010; Aceito: 11/fev./2011

\begin{abstract}
Resumo
O objetivo foi avaliar o desempenho das fórmulas DRIS na avaliação do estado nutricional de pomares de cupuaçu. Análises foliares de 153 pomares comerciais localizados no distrito de Nova Califórnia, Porto Velho, Brasil, foram usadas para comparar os diagnósticos proporcionados pelas fórmulas de Beaufils, usando-se relações bivariadas log-transformadas e adotandose o critério do Potencial de Resposta à Adubação para a interpretação dos índices DRIS. Foram avaliadas a coincidência do diagnóstico obtido pelas diferentes fórmulas, a frequência de ocorrência de cada nutriente nos diferentes tipos de estado nutricional e feita a análise multivariada para discriminar os diagnósticos entre as fórmulas adotadas. Os resultados sugerem que as fórmulas DRIS de Beaufils e de Jones são semelhantes entre si, sendo a fórmula de Wadt et al. distinta das demais, considerando-se todas as comparações realizadas entre as três fórmulas. No momento, os resultados não permitem a escoIha de qualquer uma destas fórmulas como a mais indicada para adoção em programas de recomendação de adubação.

Palavras-chave: estado nutricional, diagnose foliar, cupuaçu, frutíferas tropicais, Amazônia, Theobroma grandiflorum.
\end{abstract}

\section{DRIS functions for nutritional evaluation of cupuaçu trees}

\section{Abstract}

The objective was to evaluate the DRIS formulas performance for diagnosis of the nutritional status of cupuaçu trees. Foliar analysis of 153 commercial orchards located in the district of Nova California, Porto Velho, Brazil, were used to compare nutritional status by Beaufils formulas, using bivariate log-transformed relations and the concept of Potential Response to Fertilization for interpreting DRIS indices. The coincidence between the diagnosis by each formula, the frequency of occurrence of each nutrient in the different nutritional status and the multivariate analysis for discrimination the diagnostics between the formulas were evaluated. The results show that Beaufils and Jones formulas were similar; however, differing from Wadt et al. formula. Currently, the results do not allow the choice of one formula as the best option for adoption in fertilizer recommendation programs.

Key works: nutritional state, foliar diagnosis, tropical fruit trees, Amazon, Theobroma grandiflorum.

\section{INTRODUÇÃO}

O Sistema Integrado de Diagnose e Recomendação (DRIS) é considerado uma ferramenta promissora para a identificação de limitaçóes nutricionais que não são suficientemente diagnosticadas pelas análises de solos, como $\mathrm{N}$ e micronutrientes (Nziguheba et al., 2009), como também pela propriedade de permitir a identificação da ordem de limitação nutricional (BATAGLia et al., 2004). Trabalhos recentes com o uso deste sistema (Smithson et al., 2004; Nziguheba et al., 2009; ParTelli et al., 2006; Guindani et al., 2009; Silva et al., 2009) indicam preferência pela fórmula original de Beaufils (1973) e a simplificada de Jones (1981).
Entretanto, a escolha por uma ou outra fórmula tem sido arbitrária.

Maia (1999) sugere que a fórmula de Beaufils (1973) seria indicada apenas na avaliação de poucos nutrientes para os quais as culturas seriam mais responsivas ( $\mathrm{N}, \mathrm{P}$, e $\mathrm{K}$, por exemplo), por sua tendência em superestimar as deficiências nutricionais. Por sua vez, a fórmula de Jones (1981) não distinguiria o comportamento das plantas quanto à sua resposta à aplicação dos nutrientes, ou seja, proporciona o mesmo tratamento matemático para todos os nutrientes.

Recentemente, sugeriu-se para a cultura da mangueira, a utilização de fórmulas DRIS com capacidade de modelar o grau de dependência da cultura a 
determinados nutrientes, de modo que as fórmulas DRIS deveriam variar segundo o elemento em questão, ou para macronutriente de resposta frequente $(\mathrm{N}, \mathrm{P}$ e K, por exemplo), macronutriente de resposta rara (Ca e $\mathrm{Mg}$, por exemplo), micronutriente de resposta frequente ( $\mathrm{Zn}$, por exemplo) ou de resposta rara ou efeito tóxico ( $\mathrm{Fe}, \mathrm{Cu}$ e Mn, por exemplo) (WADT et al., 2007).

$\mathrm{Na}$ Amazônia, a domesticação dos cupuaçueiros (Theobroma grandiflorum) tem sido realizada com o cultivo de material genético de alta variabilidade fitotécnica e produtiva (Alfaia e Ayres, 2004), e em sistemas de produção com baixa adição de nutrientes ou baseadas em adubações esporádicas.

Estas condições, aliada à baixa quantidade de experimentações, têm resultado em dificuldades na definição das exigências nutricionais desta cultura e nas adubaçóes necessárias para a melhoria de sua produtividade (AlfaIA et al., 2004).

Alguns estudos têm sido realizados com o propósito de estabelecer critérios para a recomendação de adubação para o cupuaçueiro: em um ensaio em condiçóes de casa de vegetação, Fernandes et al. (2003) verificaram antagonismo entre doses elevadas de $\mathrm{P}$ e Zn, embora, a aplicação isolada dos nutrientes resultou em maior crescimento das mudas; para condiçóes de campo, Alfaia e Ayres (2004) constataram redução na produtividade com a ausência de $\mathrm{P}$ em relaçáo a aplicaçóes com $\mathrm{N}$ e $\mathrm{K}$, porém, somente para $\mathrm{K}$ foi observada resposta linear à sua aplicação e a influência do $\mathrm{N}$ foi pequena, com efeitos negativos em doses acima de $60 \mathrm{~kg} \mathrm{ha}^{-1} \mathrm{de} \mathrm{N}$.

Ayres e Alfaia (2007) apontam para a importância do nutriente potássio, uma vez que a adubação potássica isoladamente pode proporcionar um incremento na produtividade do cupuaçu de até $23 \%$, quando em dose de $80 \mathrm{~kg} \mathrm{ha}^{-1}$ de $\mathrm{K}_{2} \mathrm{O}$. A calagem, seja pelo suprimento de $\mathrm{Ca}$ e $\mathrm{Mg}$, seja pela diminuiçáo da acidez, resulta em incremento de até $27 \%$ na produtividade dos pomares (Ayres e Alfaia, 2007). Esta prática também proporciona maior absorção de outros nutrientes pelas plantas de cupuaçueiros, como $\mathrm{P}, \mathrm{Mg}, \mathrm{Ca}, \mathrm{Mn}, \mathrm{Cu}$ e $\mathrm{Zn}$ (Ayres e Alfaia, 1998).

Estas pesquisas de adubação são importantes para o desenvolvimento de sistemas de adubação para o cupuaçueiro; demandam, porém, ensaios em vários locais e por muitos anos, fazendo com que não se tenha uma rápida definição quanto ao uso da adubação na cultura. Acrescente-se que o cupuaçueiro é cultivado em diferentes situaçôes de solos, manejo cultural e sistemas de consórcios (DiAs et al. 2010), o que aumenta consideravelmente a necessidade de ensaios para que todos os fatores possam ser adequadamente modelados.

Uma alternativa consiste em adotar a análise foliar como ferramenta para apoiar as decisóes das recomendações de adubações (WADT, 2008), utilizando-se o sistema DRIS para identificar as necessidades de ajustes ou apli- caçôes de determinados nutrientes. Exige-se, entretanto, que se defina entre as diferentes fórmulas DRIS, qual representa maior potencial de utilidade para o diagnóstico nutricional de pomares de cupuaçueiros.

Neste contexto, foi objetivo deste trabalho determinar qual das fórmulas DRIS possui melhor capacidade em refletir de forma adequada o estado nutricional do cupuaçueiro para ser indicada em programas de recomendação de adubação.

\section{MATERIAL E MÉTODOS}

Entre julho e setembro de 2008, foram coletadas amostras foliares de 153 pomares comerciais de cupuaçueiro, localizados no distrito de Nova Califórnia, extremo oeste do município de Porto Velho (RO). Nestes pomares, os cupuaçueiros eram cultivados em sistemas de monocultivo (42 pomares) ou em policultivo (111 pomares), com idade variando de 5 a 18 anos. As amostras foram tomadas da terceira folha de lançamento recém-amadurecido, a partir do ápice do ramo de altura média na árvore, localizados sempre na posição norte e sul. Coletou-se um total de trinta folhas por pomar, amostrando-se de dez a quinze árvores aleatoriamente distribuídas.

O clima da região é classificado como Tropical Úmido Chuvoso - Am (Köppen), com temperatura média anual de $26^{\circ} \mathrm{C}$ e precipitação pluvial média de $2200 \mathrm{~mm}^{2}$ ano ${ }^{-1}$. O período chuvoso está compreendido entre os meses de outubro-novembro até abril-maio. No primeiro trimestre do ano observa-se o maior acúmulo de chuvas. O período mais quente fica compreendido entre agosto e outubro (SILVA, 2000).

As análises químicas das amostras foliares foram determinadas através de digestão nitro-perclórica e sulfúrica. Após a digestáo nitro-perclórica, as folhas de cupuaçu foram analisadas quanto à concentração total de cálcio $(\mathrm{Ca})$, magnésio $(\mathrm{Mg})$, manganês $(\mathrm{Mn})$, ferro $(\mathrm{Fe})$, zinco $(\mathrm{Zn})$ e cobre $(\mathrm{Cu})$ por espectrometria de plasma (ICP-OES), potássio $(\mathrm{K})$ por fotometria de chama e fósforo $(\mathrm{P})$ por espectrofotometria molecular. O nitrogênio $(\mathrm{N})$ total foi obtido após digestão sulfúrica e destilação por Kjehdahl (CARmo et al., 2000). Os nutrientes N, P, K, Ca e Mg foram expressos em $\mathrm{g} \mathrm{kg}^{-1}$ e os nutrientes $\mathrm{Zn}, \mathrm{Fe}, \mathrm{Mn}$ e $\mathrm{Cu}$, em $\mathrm{mg} \mathrm{kg}^{-1}$.

As normas DRIS foram obtidas a partir do conjunto da populaçáo monitorada, realizando a transformação logarítmica das relaçóes bivariadas (BEvERLy, 1993), e calculando-se a média e variância das respectivas relaçóes logtransformadas. A utilização de todos os pomares para a obtenção das normas DRIS e a transformação logarítmica das relaçôes nutricionais bivariadas traz como benefícios maior representatividade, melhor estimativa dos parâmetros populacionais e maior consistência dos dados, aliado ao fato de que o uso de diferentes normas DRIS para 
cupuaçu, envolvendo pomares em monocultivo ou consórcio e de diferentes idades, indicou o mesmo desempenho na identificaçáo do estado nutricional dos pomares.

As fórmulas DRIS testadas foram: fórmula original ou de Beaufils (Beaufils, 1973), fórmula simplificada ou de Jones (Jones, 1981) e a fórmula para modelagem das funçôes DRIS ou de WADT et al. (WADT et al., 2007; WADT e SILVA, 2010). Todas as fórmulas foram adaptadas para o uso de relaçôes log-transformadas (WADT e SiLVA, 2010) e foram utilizadas no cálculo dos índices DRIS todas as relaçôes, na forma direta e inversa:

Inut $=\left[\mathrm{f}\left(\mathrm{A} / \mathrm{B}_{1}\right)+\mathrm{f}\left(\mathrm{A} / \mathrm{B}_{2}\right)+\ldots+\mathrm{f}\left(\mathrm{A} / \mathrm{B}_{\mathrm{n}}\right)+\ldots-\mathrm{f}\left(\mathrm{B}_{1} / \mathrm{A}\right)-\mathrm{f}\left(\mathrm{B}_{2} / \mathrm{A}\right)-\ldots . \mathrm{f}\left(\mathrm{B}_{\mathrm{n}} / \mathrm{A}\right)\right] /(2 \mathrm{n}) \mathrm{e}$;

A transformação logarítmica das relações bivariadas proposta por BeVERLY (1987) tem como objetivo corrigir os desvios observados na distribuição normal entre as formas diretas e inversas de uma mesma relação nutricional.

Dada uma relaçáo para quaisquer dois nutrientes " $R$ " e "S", cujas concentraçôes médias sejam expressas em $[\mathrm{r}]$ e [s], quando $\mathrm{R}>\mathrm{S}$, observa-se constantemente que $[\mathrm{r}] /[\mathrm{s}]>$ $1 /[\mathrm{r}] /[\mathrm{s}]$. Este comportamento consiste em uma fraqueza na distribuição dos valores das relações bivariadas, uma vez que formas alternativas de expressão deveriam resultar em medidas equivalentes para o balanço entre os nutrientes. Esta falha introduz erros sistemáticos na abordagem tradicional.

Com a transformaçáo logarítmica proposta por BEVERLY (1987), esta anomalia na distorção não é observada, uma vez que $\log [\mathrm{r}] /[\mathrm{s}]=-\log 1 /([\mathrm{r}] /[\mathrm{s}]$, ou seja, para qualquer valor de "R" e "S", ambas as formas de expressão resultam em desvios de mesma magnitude.

As fórmulas, log-transformadas, ficaram sendo:

1) Fórmula original de Beaufils (1973), com relaçóes log-transformadas:

Quando $\log (\mathrm{A} / \mathrm{B})<\log (\mathrm{a} / \mathrm{b})$, então:

$\mathrm{f}(\mathrm{A} / \mathrm{B})=\left\{[\log (\mathrm{A} / \mathrm{B})-\log (\mathrm{a} / \mathrm{b})] / \sigma_{(\log (a / b))}\right\} \times[\log (\mathrm{a} / \mathrm{b}) / \log (\mathrm{A} / \mathrm{B})$

Senão:

$f(A / B)=\left\{[\log (A / B)-\log (a / b)] / \sigma_{(\log (a / b))}\right\}$

2) Fórmula simplificada de Jones (1981), com relações log-transformadas:

$\mathrm{f}(\mathrm{A} / \mathrm{B})=\left\{[\log (\mathrm{A} / \mathrm{B})-\log (\mathrm{a} / \mathrm{b})] / \sigma_{(\log (\mathrm{a} / \mathrm{b})}\right\}$

3) Fórmula para modelagem das funçóes DRIS (MAIA, 1999; WADt et al., 2007; WADt e Silva, 2010) colaboradores, com relaçóes log-transformadas:

Quando o elemento "A" for os nutrientes N, P ou K, então: $\mathrm{f}(\mathrm{A} / \mathrm{B})=\mathrm{fk} \times\left\{[\log (\mathrm{A} / \mathrm{B})-\log (\mathrm{a} / \mathrm{b})] / \sigma_{(\log (\mathrm{a} / \mathrm{b})}\right\} \times[\log (\mathrm{a} / \mathrm{b})$

Quando o elemento "A" for os nutrientes $\mathrm{Ca}$ ou $\mathrm{Mg}$, se $\log (\mathrm{A} / \mathrm{B})<\log (\mathrm{a} / \mathrm{b})$ e $\log (\mathrm{A} / \mathrm{B})>\log (\mathrm{a} / \mathrm{b}) / 2$, então:
$\mathrm{f}(\mathrm{A} / \mathrm{B})=\mathrm{fk} x\left\{[\log (\mathrm{A} / \mathrm{B})-\log (\mathrm{a} / \mathrm{b})] / \sigma_{(\log (a / b)}\right\} \times[\log (\mathrm{A} / \mathrm{B}) / \log (\mathrm{a} / \mathrm{b})]$

se $\log (\mathrm{A} / \mathrm{B})<\log (\mathrm{a} / \mathrm{b})$ e $\log (\mathrm{A} / \mathrm{B})<=\log (\mathrm{a} / \mathrm{b}) / 2$, entâo:

$\mathrm{f}(\mathrm{A} / \mathrm{B})=\mathrm{fkx}\left\{[\log (\mathrm{A} / \mathrm{B})-\log (\mathrm{a} / \mathrm{b})] / \sigma_{(\log (\mathrm{a} / \mathrm{b})}\right\}$

se $\log (\mathrm{A} / \mathrm{B})>=\log (\mathrm{a} / \mathrm{b})$, então:

$\mathrm{f}(\mathrm{A} / \mathrm{B})=\mathrm{fk} x\{[\log (\mathrm{A} / \mathrm{B})-\log (\mathrm{a} / \mathrm{b})] / \sigma(\log (\mathrm{a} / \mathrm{b}))\} \times[\log (\mathrm{a} / \mathrm{b}) / \log (\mathrm{A} / \mathrm{B})]$

Quando o elemento "A" for o nutriente $\mathrm{Zn}$,

se $\log (\mathrm{A} / \mathrm{B})<\log (\mathrm{a} / \mathrm{b})$, então:

$\mathrm{f}(\mathrm{A} / \mathrm{B})=\mathrm{fk} \times\left\{[\log (\mathrm{A} / \mathrm{B})-\log (\mathrm{a} / \mathrm{b})] / \sigma_{(\log (\mathrm{a} / \mathrm{b}))}\right\}$

se $\log (\mathrm{A} / \mathrm{B})>=\log (\mathrm{a} / \mathrm{b})$, então:

$\mathrm{f}(\mathrm{A} / \mathrm{B})=\mathrm{fk} x\left\{[\log (\mathrm{A} / \mathrm{B})-\log (\mathrm{a} / \mathrm{b})] / \sigma_{(\log (\mathrm{a} / \mathrm{b})}\right\} \mathrm{x}[\log (\mathrm{A} / \mathrm{B}) / \log (\mathrm{a} / \mathrm{b})]$

Quando o elemento "A" for o nutriente $\mathrm{Fe}, \mathrm{Mn}$ ou $\mathrm{Cu}$, se $\log (\mathrm{A} / \mathrm{B})<\log (\mathrm{a} / \mathrm{b})$ e $\log (\mathrm{A} / \mathrm{B})>\log (\mathrm{a} / \mathrm{b}) / 2$, então:

$\mathrm{f}(\mathrm{A} / \mathrm{B})=\mathrm{fk} x\left\{[\log (\mathrm{A} / \mathrm{B})-\log (\mathrm{a} / \mathrm{b})] / \sigma_{(\log (/ \mathrm{b}) \mathrm{b}}\right\} \times[\log (\mathrm{A} / \mathrm{B}) / \log (\mathrm{a} / \mathrm{b})]$

se $\log (\mathrm{A} / \mathrm{B})<\log (\mathrm{a} / \mathrm{b})$ e $\log (\mathrm{A} / \mathrm{B})<=\log (\mathrm{a} / \mathrm{b}) / 2$, entâo:

$\mathrm{f}(\mathrm{A} / \mathrm{B})=\mathrm{fk} \times\left\{[\log (\mathrm{A} / \mathrm{B})-\log (\mathrm{a} / \mathrm{b})] / \sigma_{(\log (a / b))}\right\}$

se $\log (A / B)>=\log (a / b)$, então:

$\mathrm{f}(\mathrm{A} / \mathrm{B})=\mathrm{fkx}\left\{[\log (\mathrm{A} / \mathrm{B})-\log (\mathrm{a} / \mathrm{b})] / \sigma_{(\log (\mathrm{a} / \mathrm{b})}\right\}$

Sendo: Inut: índice nutricional;

$\mathrm{f}(\mathrm{A} / \mathrm{B})$ corresponde à função DRIS para dois nutrientes ( $\mathrm{A}$ e $\mathrm{B}$ ) quaisquer; $\mathrm{n}$, correspondente ao número de nutrientes analisados; $\mathrm{B}_{1}, \mathrm{~B}_{2}, \mathrm{~B}_{\mathrm{n}}$, corresponde ao nutrientes relacionados ao nutriente $\mathrm{A} ; \log (\mathrm{A} / \mathrm{B})$ corresponde à relação entre os nutrientes $\mathrm{A}$ e $\mathrm{B}$ na amostra; $\log (\mathrm{a} / \mathrm{b})$ corresponde à relação entre os nutrientes A e B na norma de referência;

$\sigma_{(\log (a / b))}$ corresponde ao desvio-padrão da relação A e B na norma de referência e;

$\mathrm{fk}$ foi igual a 2 para os nutrientes $\mathrm{N}, \mathrm{P}, \mathrm{K}$; foi igual a 1,5 para os nutrientes $\mathrm{Zn}$ e $\mathrm{Mn}$; igual a 1 para o nutriente $\mathrm{Fe}$ e; igual a 0,5 para os nutrientes, $\mathrm{Ca}$ e $\mathrm{Mg}$ e $\mathrm{Cu}$.

$\mathrm{O}$ índice de balanço nutricional médio (IBNm) foi calculado pela média aritmética do somatório dos valores absolutos dos índices nutricionais (Inut) gerados para cada amostra.

Para a interpretação do estado nutricional, utilizouse o critério do Potencial de Resposta à adubaçáo (WADT, 2005), sendo os nutrientes classificados em cinco categorias:

i) Equilíbrio nutricional $=\mid$ Inut $\mid<\mathrm{IBNm}$. Atribuiu-se o valor zero para esta condição;

ii) Alta probabilidade de insuficiência $\mid$ Inut $\mid>$ IBN, Inut $<0$ e Inut sendo o de menor valor. Atribui-se o valor -2 para esta condição; 
iii) Baixa probabilidade de insuficiência $\mid$ Inut $\mid>$ IBN, Inut $<0$ e Inut não sendo o de menor valor. Atribuise o valor -1 para esta condição;

iv) Alta probabilidade de excesso $\mid$ Inut $\mid>\mathrm{IBNm}$, Inut $>$ 0 e Inut sendo o de maior valor. Atribui-se o valor 2 para esta condição; e

v) Baixa probabilidade de excesso $\mid$ Inut $\mid>$ IBNm, Inut $>0$ e Inut não sendo o de maior valor. Atribui-se o valor 1 para esta condiçáo

Para a comparação dos diagnósticos produzidos entre as três fórmulas DRIS testadas, os nutrientes foram classificados em três categorias: insuficientes, equilibrados ou em excesso, sem distinção da probabilidade de ocorrência de determinado estado nutricional.

Os índices DRIS dos nutrientes e a classificaçáo quanto ao potencial de resposta à adubaçáo de cada fórmula DRIS foram os parâmetros utilizados para se avaliar o desempenho comparativo entre as funçóes, utilizandose a análise discriminante multivariada.

Cada uma das interpretaçóes obtidas, para cada nutriente e pomar, foram comparadas entre si com os critérios obtidos pelas diferentes fórmulas utilizadas, anotando-se o valor 1 quanto houve coincidência no diagnóstico do estado nutricional (ou seja, para um mesmo pomar e nutriente, dois métodos indicavam o mesmo estado nutricional) e o valor 0 quando não houve esta coincidência no diagnóstico. As análises de contagem das coincidências nos diferentes diagnósticos obtidos foram feitos em planilha eletrônica (Calc do OpenOffice.org), computando-se a porcentagem de casos coincidentes para a comparação das fórmulas DRIS duas a duas.

Os índices DRIS, IBNm e a interpretação do potencial de resposta à adubação, foram obtidos por meio do software DRIS Cupuaçu (www.dris.com.br), o qual consiste de um sistema web desenvolvido em linguagem de programação $\mathrm{PHP}$ e com sistema gestor de banco de dados MySQL, com interface para inclusão, edição e exclusão de dados de análises foliares, geração de normas DRIS e cálculos dos índices DRIS por diferentes fórmulas ou procedimentos de tratamento dos dados.

Utilizou-se a análise multivariada discriminante (discriminant analysis) para construir modelos preditivos capazes de distinguir os pomares quanto ao diagnóstico nutricional proporcionado pelas diferentes fórmulas DRIS. Os modelos foram compostos pelas duas principais funçôes discriminantes, obtidas da combinaçáo linear dos índices DRIS ou dos valores atribuídos ao estado nutricional pelo método do potencial de resposta à adubação. As funções discriminantes obtidas foram usadas para reclassificar os dados, verificando a capacidade destas em distinguir os pomares quanto às fórmulas utilizadas.

Quando a reclassificação foi incorreta, denominou-se de confusão entre as fórmulas DRIS, significando que há muita semelhança entre as fórmulas; quando a reclassificação foi correta, implicou que as fórmulas DRIS resultam em diagnósticos distintos. A porcentagem de diagnósticos corretamente reclassificados foi apresentada na forma de uma matriz de confundimento. Estas análises multivariadas foram realizadas pelo software SPSS 16.0 (SPSS, 2009).

Realizou-se também análise da frequência dos nutrientes quanto ao estado nutricional, contando-se o número de pomares de cupuaçueiros, para cada nutriente, com um dos cinco possíveis estados nutricionais: insuficiência com alta probabilidade (IAP), insuficiência com baixa probabilidade (IBP), equilibrado (EQU), excesso com baixa probabilidade (EBP) ou excesso com alta probabilidade (EAP).

\section{RESULTADOS E DISCUSSÃO}

A fórmula de cálculo para os índices DRIS para macronutrientes não responsivos $(\mathrm{Ca}$ e $\mathrm{Mg}$ ), sugerida por WADT et al. (2007), representa um erro conceitual na avaliação da insuficiência, pois quando o valor da relação nutricional for menor que a metade do valor da norma, o valor da função DRIS que deveria diminuir (afastar-se do equilíbrio), passa a aumentar (aproximar-se do equilíbrio). Esse processo decorre da adoção de uma equação quadrática, com uma tangente decrescente para cada ponto da curva entre a o valor da norma e o ponto de mínimo, para depois esta tangente tornar-se crescente para valores menores que o ponto de mínimo. Para corrigir este erro na fórmula, o fator $\log (\mathrm{A} / \mathrm{B}) / \log (\mathrm{a} / \mathrm{b})$ somente foi utilizado enquanto o valor $\log (\mathrm{A} / \mathrm{B})$ for menor que $\log (\mathrm{a} / \mathrm{b}) / 2$, fazendo com que a partir do ponto de inflexão da curva (ponto de mínimo), a equação seja linear e decrescente.

$\mathrm{Na}$ análise de agrupamento dos pomares por meio das duas principais funções discriminantes obtidas pelos índices DRIS das diferentes fórmulas utilizadas, notou-se que não houve distinção entre as fórmulas de JonEs (1981) e de Beaufils (1973) (Figura 1), enquanto a fórmula de WADT et al. (2007) resultou em um grupamento distinto. Ao se utilizar para a reclassificação o valor atribuído ao Potencial de Resposta à Adubaçáo (números inteiros de -2 $a+2)$, as diferenças entre as fórmulas tenderam a se anular (Figura 2).

A reclassificação dos pomares com base no estado nutricional determinado pelas diferentes fórmulas DRIS indica, entre as fórmulas de Jones (1981) e BeAufiLs (1973), que há confusão nos diagnósticos gerados, ou seja, não é possível distinguir os pomares quanto ao estado nutricional indicado por qualquer uma destas fórmulas pelo alto grau de semelhança entre os diagnósticos resultantes, conforme se observa na matriz de confundimento. Por sua vez, na fórmula de WADT et al. (2007), distingue-se das demais (Tabela 1). 


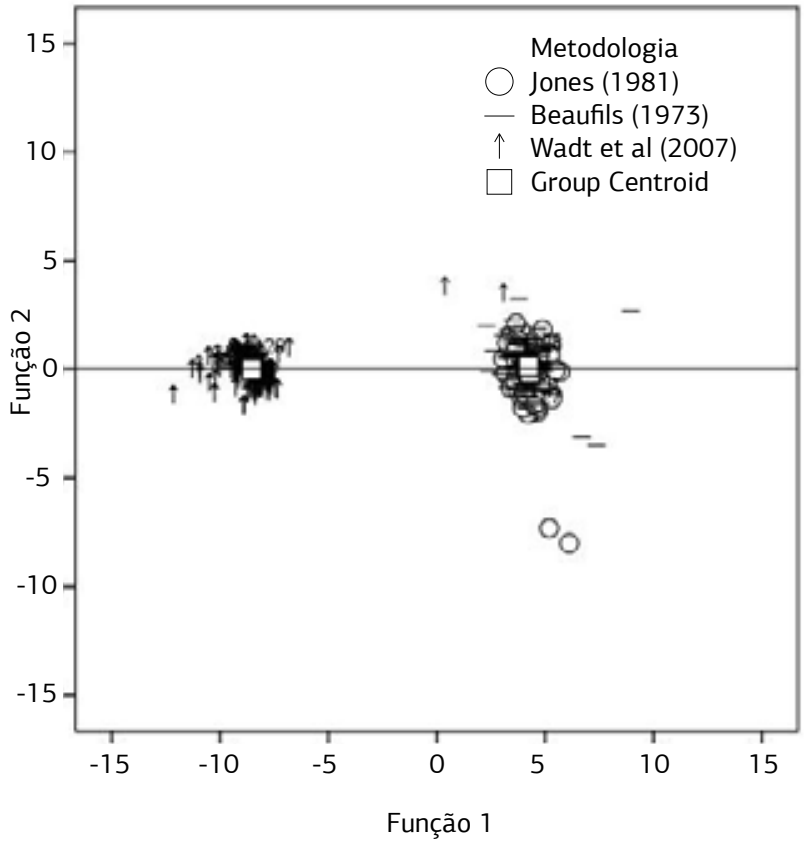

Figura 1. Diagrama de dispersão para os pomares de cupuaçu em função das duas principais funçôes discriminantes quanto aos índices DRIS obtidos pelas fórmulas de Jones (1981), BEAUfILS (1973) e de WADT et al. (2007).

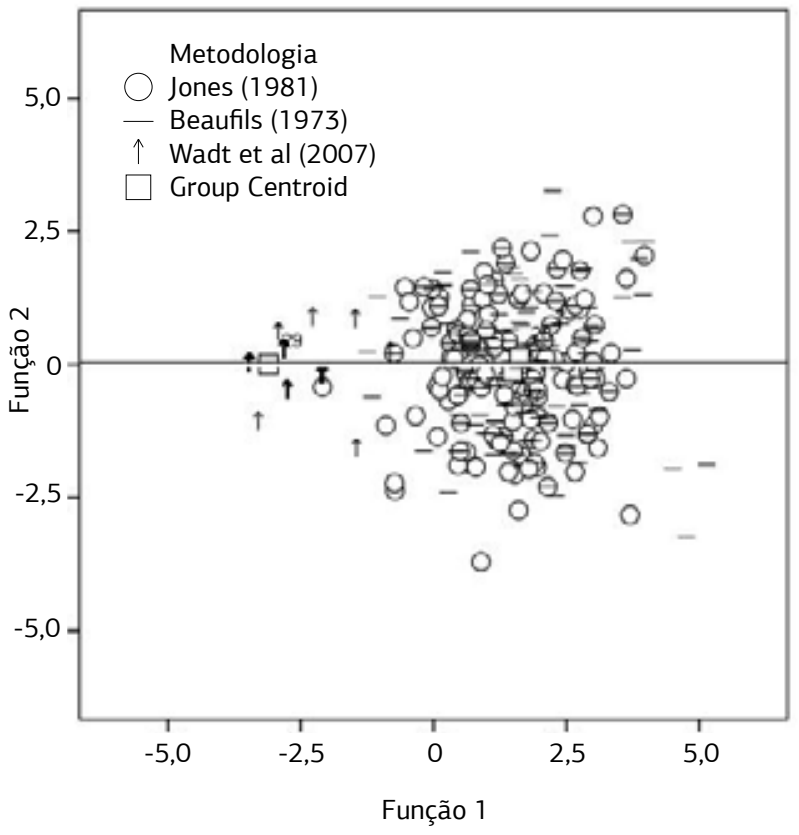

Figura 2. Diagrama de dispersão para os pomares de cupuaçu em função das duas principais funçóes discriminantes quanto ao potencial de resposta à adubação, obtidas pelas fórmulas de JoNES (1981), Beaufils (1973) e Wadt et al. (2007).

Tabela 1. Porcentagem do número de casos (pomares) classificados corretamente e incorretamente para cada grupo obtido pela análise discriminante

\begin{tabular}{|c|c|c|c|c|}
\hline & & \multicolumn{3}{|c|}{ Grupamentos preditos } \\
\hline & & JONES (1981) & BeAufiLs (1973) & WADT et al. (2007) \\
\hline \multirow{8}{*}{ Grupamentos reais } & & \multicolumn{3}{|c|}{ Com base no potencial de resposta à adubação } \\
\hline & JONES (1981) & 52 & 46 & 1 \\
\hline & BEAUFILS (1973) & 46 & 52 & 2 \\
\hline & WADT et al. (2007) & 0 & 1 & 99 \\
\hline & & \multicolumn{3}{|c|}{ Com base nos valores dos índices DRIS } \\
\hline & JONES (1981) & 52 & 48 & 0 \\
\hline & BEAUFILS (1973) & 42 & 58 & 0 \\
\hline & WADT et al. (2007) & 0 & 1 & 99 \\
\hline
\end{tabular}

Fazendo-se para cada nutriente a contagem dos diagnósticos obtidos em cada um dos estados nutricionais possíveis, as fórmulas de Jones (1981) e de BeaufiLs (1973) novamente indicam elevado grau de concordância, o qual variou de $85,0 \%$ (para Ca) até 96,1\% (para Fe). Por sua vez, na fórmula de WADT et al. (2007), quando comparada com as demais, o grau de concordância foi sempre inferior a $64 \%$, atingindo para o $\mathrm{N}$ apenas $12,4 \%$ quando comparada com a de Beaufils (1973) (Tabela 2).

Por outro lado, ao se analisar a frequência com que os nutrientes são classificados como insuficientes, equilibrados ou em excesso, observa-se que esta frequência é relativamente uniforme para todos os nutrientes, quando se utiliza a fórmula de Jones (1981) ou BeAufILs (1973); a fórmula de WADT et al. (2007) tende a indicar determi- nados nutrientes como potencialmente desequilibrados e outros, como equilibrados (Tabela 3).

Para N, por exemplo, a fórmula de WADT et al. (2007) indicou 151 casos de insuficiência, sendo destes, 114 com alta probabilidade. Para as outras fórmulas, o número de casos de $\mathrm{N}$ com insuficiência foi de 24 para a fórmula de Jones (1981) e de 18 para a de Beaufils (1973). P e $\mathrm{K}$ não foram considerados limitantes para a nutrição de cupuaçueiros pela fórmula de WADT et al. (2007), onde houve somente um pomar com indicação de insuficiência para cada um destes elementos. Por sua vez, as demais fórmulas indicaram 32 a 33 pomares com insuficiência de $\mathrm{P}$ e de 34 a 41 pomares com insuficiência de $\mathrm{K}$.

De modo semelhante, $\mathrm{Ca}$ e $\mathrm{Mg}$ foram suficientes com base na fórmula de WADT et al. (2007), com um único 
caso de insuficiência para $\mathrm{Ca}$ e nenhum para $\mathrm{Mg}$, enquanto pelas demais fórmulas, o número de pomares com insuficiência variou de 30 a 33 para Ca e de 19 a 21 para $\mathrm{Mg}$.

A deficiência de $\mathrm{P}$ e $\mathrm{K}$ em solos sob cultivo de cupuaçu na Região Amazônica tem sido demonstrada (AlfaiA et al., 2004), observando-se respostas, mesmo em Cambissolos, à aplicação de P (Alfaia e Ayres, 2004). Considerando-se que os pomares monitorados não receberam

Tabela 2. Porcentagem do número de diagnósticos nutricionais semelhantes obtido pelas diferentes fórmulas DRIS, comparadas duas a duas

\begin{tabular}{|cccc|}
\hline Nutriente & $\begin{array}{c}\text { Jones } \mathbf{x} \\
\text { Beaufils }\end{array}$ & $\begin{array}{c}\text { Jones } \mathbf{x} \\
\text { Wadt et al. }\end{array}$ & $\begin{array}{c}\text { Beaufils } \mathbf{x} \\
\text { Wadt et al. }\end{array}$ \\
\hline $\mathrm{N}$ & 92,2 & 15,7 & 12,4 \\
\hline $\mathrm{P}$ & 90,8 & 18,3 & 20,3 \\
\hline $\mathrm{K}$ & 88,9 & 47,7 & 52,9 \\
\hline $\mathrm{Ca}$ & 85,0 & 49,7 & 56,9 \\
\hline $\mathrm{Mg}$ & 87,6 & 63,4 & 57,5 \\
\hline $\mathrm{Zn}$ & 88,2 & 38,6 & 38,6 \\
\hline $\mathrm{Fe}$ & 96,1 & 24,8 & 24,8 \\
\hline $\mathrm{Mn}$ & 90,2 & 35,3 & 33,3 \\
\hline $\mathrm{Cu}$ & 90,2 & 44,4 & 50,3 \\
\hline
\end{tabular}

qualquer tipo de adubação e os experimentos de AlfaIA e Ayres (2004) indicam deficiência para P, a fórmula de WADT et al. (2007) superestimou a condiçáo de excesso para este nutriente.

Deve-se considerar, entretanto, que o fato de P propiciar elevada mobilidade entre folhas novas e velhas (SALVADOR et al., 1994) e a presença de micorrizas pode contribuir para maior eficiência do fósforo nativo, é possível que a baixa variabilidade nos teores de $\mathrm{P}$ nas amostras tenha contribuído para este diagnóstico fornecido pela fórmula de WADT et al. (2007).

Com relaçáo ao $\mathrm{K}$, experimentos realizados sobre Cambissolos localizados no distrito de Nova Califórnia indicam resposta positiva a este nutriente, incrementando a produtividade do cupuaçueiro em até $23 \%$ e resposta linear até a dose de $80 \mathrm{~kg} \mathrm{ha}^{-1}$ de $\mathrm{K}_{2} \mathrm{O}$ (Ayres e Alfaia, 2007; Alfaia e Ayres, 2004). Entretanto a fórmula de WADT et al. (2007) não conseguiu identificar a insuficiência de K para cupuaçueiros, uma vez que indicou este nutriente em condição de equilíbrio nutricional na maioria dos pomares avaliados.

Para N, Alfaia e Ayres (2004) relatam resposta negativa quanto à produtividade com a adiçáo deste nutriente

Tabela 3. Contagem do número de pomares de cupuaçueiro com estados nutricionais de insuficiência com alta probabilidade, (IAP) insuficiência com baixa probabilidade (IBP), equilibrados, (EQU) excesso com baixa probabilidade (EBP) e excesso com alta probabilidade (EAP), em relação a macro e micronutrientes

\begin{tabular}{|c|c|c|c|c|c|c|}
\hline \multirow{2}{*}{$\begin{array}{l}\text { Fórmula } \\
\text { utilizada }\end{array}$} & \multirow[t]{2}{*}{ Nutriente } & \multicolumn{5}{|c|}{ Provável estado nutricional } \\
\hline & & IAP & IBP & EQU & EBP & EAP \\
\hline Jones & $\mathrm{N}$ & 6 & 18 & 112 & 12 & 5 \\
\hline Beaufils & $\mathrm{N}$ & 3 & 15 & 113 & 15 & 7 \\
\hline Wadt et al. & $\mathrm{N}$ & 114 & 37 & 1 & 0 & 1 \\
\hline Jones & $\mathrm{P}$ & 12 & 20 & 94 & 17 & 10 \\
\hline Beaufils & $\mathrm{P}$ & 11 & 22 & 90 & 19 & 11 \\
\hline Wadt et al. & $\mathrm{P}$ & 0 & 1 & 1 & 1 & 150 \\
\hline Jones & $\mathrm{K}$ & 11 & 30 & 70 & 22 & 20 \\
\hline Beaufils & $\mathrm{K}$ & 8 & 26 & 79 & 22 & 18 \\
\hline Wadt et al. & $\mathrm{K}$ & 0 & 1 & 149 & 2 & 1 \\
\hline Jones & $\mathrm{Ca}$ & 15 & 18 & 76 & 31 & 13 \\
\hline Beaufils & $\mathrm{Ca}$ & 11 & 19 & 87 & 23 & 13 \\
\hline Wadt et al. & $\mathrm{Ca}$ & 0 & 0 & 153 & 0 & 0 \\
\hline Jones & $\mathrm{Mg}$ & 9 & 12 & 97 & 23 & 12 \\
\hline Beaufils & $\mathrm{Mg}$ & 9 & 10 & 88 & 30 & 16 \\
\hline Wadt et al. & $\mathrm{Mg}$ & 0 & 0 & 153 & 0 & 0 \\
\hline Jones & $\mathrm{Zn}$ & 12 & 26 & 88 & 15 & 12 \\
\hline Beaufils & $\mathrm{Zn}$ & 16 & 23 & 90 & 14 & 10 \\
\hline Wadt et al. & $\mathrm{Zn}$ & 0 & 125 & 28 & 0 & 0 \\
\hline Jones & $\mathrm{Fe}$ & 32 & 43 & 38 & 12 & 28 \\
\hline Beaufils & $\mathrm{Fe}$ & 47 & 30 & 38 & 11 & 27 \\
\hline Wadt et al. & $\mathrm{Fe}$ & 0 & 0 & 153 & 0 & 0 \\
\hline Jones & $\mathrm{Mn}$ & 28 & 26 & 44 & 18 & 37 \\
\hline Beaufils & $\mathrm{Mn}$ & 28 & 22 & 45 & 22 & 36 \\
\hline Wadt et al. & $\mathrm{Mn}$ & 39 & 113 & 1 & 0 & 0 \\
\hline Jones & $\mathrm{Cu}$ & 28 & 12 & 68 & 29 & 16 \\
\hline Beaufils & $\mathrm{Cu}$ & 20 & 19 & 77 & 22 & 15 \\
\hline Wadt et al. & $\mathrm{Cu}$ & 0 & 0 & 153 & 0 & 0 \\
\hline
\end{tabular}


para cupuaçueiros sob monocultivo, decorrente do efeito benéfico da ciclagem de nutrientes sobre a disponibilidade de N no solo (Schwendener et al., 2005). Por este motivo, o diagnóstico nutricional proporcionado pelas fórmulas de BEAUfils (1973) e Jones (1981) foram mais coerentes que aquele proporcionado pela fórmula de WADT et al. (2007), a qual indicou para a maioria dos pomares insuficiência de $\mathrm{N}$.

Para os micronutrientes, a fórmula de WADT et al. (2007) indicou o Mn (152 pomares) e o Zn (125 pomares) como nutrientes responsivos à adubaçáo; para estes nutrientes, as demais fórmulas indicaram de 50 a 54 pomares com insuficiência para Mn e de 38 a 39 pomares com insuficiência para $\mathrm{Zn}$.

Quanto ao $\mathrm{Fe}$ e $\mathrm{Cu}$, enquanto as fórmulas convencionais revelam grande número de casos de insuficiência (75 a 77 casos para $\mathrm{Fe}$ e 39 a 40 casos para $\mathrm{Cu}$ ), a fórmula de WADT et al. (2007) não indicou um único caso de insuficiência .

Não há na literatura resultados conclusivos sobre as demandas de micronutrientes ou de $\mathrm{Ca}$ e $\mathrm{Mg}$ para o cupuaçueiro; a única pesquisa com micronutrientes indica resposta de mudas de cupuaçueiros à adição de $\mathrm{Zn}$, na ausência de P (Fernandes et al., 2003). Também, Ayres e Alfaia (2007) relatam a importância da calagem para incrementar a produtividade do cupuaçueiro como também para melhorar a eficiência do $\mathrm{P}$ e a disponibilidade de K nesta cultura.

O número de casos de deficiência para cada um dos nutrientes varia, para as fórmulas de Jones (1981) e BEAufiLs (1973) em torno de 38,7 casos (21 para N, 32,5 para $\mathrm{P}, 37,5$ para $\mathrm{K}, 31,5$ para $\mathrm{Ca}, 20$ para $\mathrm{Mg}, 38,5$ para $\mathrm{Zn}, 76$ para $\mathrm{Fe}, 52$ para $\mathrm{Mn}$ e 39,5 para $\mathrm{Cu}$ ), independentemente da importância relativa dos nutrientes para a nutrição dos pomares. Estas fórmulas tendem a distribuir as insuficiências em frequências relativamente aproximadas entre todos os nutrientes avaliados, fato não suportado nos ensaios de adubação realizados com esta cultura. Esta distribuição sugere alguma aleatoriedade na avaliação do estado nutricional, necessitando-se mais estudos para definir sobre a indicação de alguma destas fórmulas em programas de adubação.

De modo distinto, a fórmula de WADT et al. (2007) indicou somente três nutrientes como insuficientes (151 pomares para N, 152 pomares para Mn e 125 pomares para $\mathrm{Zn}$ ), sendo os demais nutrientes, considerados não limitantes nas condições atuais de manejo dos pomares. Este resultado é contraditório com a literatura especializada, onde $\mathrm{N}$ é normalmente apontado como deficiente $\mathrm{e}$ há respostas à adição de $\mathrm{P}$ e K.

Dado que a fórmula de WADT et al. (2007) possibilita o ajuste do coeficiente de sensibilidade (WADt e Lemos, 2010), há possibilidades de que resultados mais promissores possam ser alcançados com a modelagem das funções DRIS (WaDt e Silva, 2010).
As fórmulas DRIS testadas (BeAufiLs, 1973; Jones, 1981; WADT et al., 2007) carecem de avaliações mais detalhadas para que possam ser utilizadas em programas de adubação para o cupuaçueiro.

\section{CONCLUSÃO}

As fórmulas de Beaufils (1973) e Jones (1981) possuem capacidades semelhantes em realizar os diagnósticos do estado nutricional de cupuaçueiros, sendo a fórmula de WADT et al. (2007) distinta das demais. Novos estudos são necessários para que se possa recomendar uma destas fórmulas como a mais indicada para utilização em programas de recomendaçáo de adubação para o cupuaçueiro.

\section{AGRADECIMENTOS}

Ao CNPq e à FUNTAC, pelo apoio financeiro ao projeto de pesquisa, e aos produtores e técnicos do Projeto de Reflorestamento Econômico Consorciado Adensado (RECA), de Nova Califórnia, Porto Velho (RO), pelo apoio logístico e colaboração nos trabalhos de campo.

\section{REFERÊNCIAS}

ALFAIA, S.S.; AYRES, M.I.C. Efeito de doses de nitrogênio, fósforo e potássio em duas cultivares de cupuaçu, com e sem semente, na regiâo da Amazônia Central. Revista Brasileira de Fruticultura, v.26, p.320-325, 2004.

ALFAIA, S.S.; RIBEIRO, G.A.; NOBRE, A.D.; LUIZÃO, R.C.; LUIZÃO, F.J. Evaluation of soil fertility in smallholder agroforestry systems and pastures in western Amazonia. Agriculture, Ecosystems and Environment, v.102, p.409-414, 2004.

AYRES, M.I.C.; ALFAIA, S.S. Calagem e adubação potássica na produção do cupuaçuzeiro em sistemas agroflorestais da Amazônia ocidental. Pesquisa Agropecuária Brasileira, v.42, p.957-963, 2007.

AYRES, M.I.C.; ALFAIA, S.S. Efeito de NPK, calagem e micronutrientes na produção de frutos do cupuaçuzeiro. Revista Brasileira de Fruticultura, v.32, p.863-870, 1998.

BEAUFILS, E.R. Diagnosis and recommendation integrated system (DRIS). Pietermaritzburg: University of Natal, 1973, 132 p. (Soil Science Bulletin, 1)

BEVERLY, R.B. Comparison of DRIS and alternative nutrient diagnostic methods for soybean. Journal of Plant Nutrition, v.10, p.901-920, 1987.

BEVERLY, R. B. DRIS Diagnoses of soybean nitrogen, phosphorus, and potassium status are unsatisfactory. Journal of Plant Nutrition, v.16, p.1431-1447, 1993. 
CARMO, C.A.F.S.; ARAÚJO, W.S.; BERNARDI, A.C.C.; SALDANHA, M.F.C. 2000. Métodos de análise de tecidos vegetais utilizados pela Embrapa Solos. Rio de Janeiro: Embrapa Solos, 2000. 41p.

DIAS, J.R.M.; PEREZ, D.V.; LEMOS, C.O.; SILVA, L.M.; WADT, P.G.S. Relaçóes nutricionais log-transformadas para avaliação nutricional de cupuaçueiros comerciais. Acta Amazonica, v.40, p.37-40, 2010.

FERNANDES, A.R.; CARVALHO, J.G.; MELO, P.C. Efeito do fósforo e do zinco sobre o crescimento de mudas do cupuaçuzeiro (Theobroma grandiflorum schum.). Revista Cerne, v.9, p.221-230, 2003.

GUINDANI, R.R.H.P.; ANGHINONI, I.; NACHTIGALL, G.R. DRIS na avaliaçáo do estado nutricional do arroz irrigado por inundação. Revista Brasileira de Ciência do Solo, v.33, p.109-118, 2009.

JONES, W.W. Proposed modifications of the diagnosis and recommendation integrated system (DRIS) for interpreting plant analyses. Communications in Soil Science and Plant Analysis, v.12, p.785-794, 1981 .

MAIA, C. E. Análise crítica da fórmula original de Beaufils no cálculo dos índices DRIS: a constante de sensibilidade. In: WADT, P.G.S.; MALAVOLTA, E. (Ed.). Monitoramento nutricional para a recomendação de adubação para as culturas. Piracicaba: POTAFÓS, 1999. 17p. (CD-ROM)

NIZGUHEBA, G.; TOSSAH, B.K.; DIELS, J.; FRANKE, A.C.; AIHOU, K.; IWUAFOR, E.N.O; NWOKE, C.; MERCKX, R.. Assessment of nutrient deficiencies in maize in nutrient omission trials and long-term field experiments in the West African Savanna. Plant and Soil, v. 314, p.143-157, 2009.

PARTELLI, F.L.; VIEIRA, H.D.; MONNERAT, P.H., VIANA, A.P. Estabelecimento de normas DRIS em cafeeiro conilon orgânico e convencional no Estado do Espírito Santo. Revista Brasileira de Ciência do Solo, v.30, p.20-25, 2006.

SALVADOR, J.O.; MURAOKA, T.; ROSSETO, R.; RIBEIRO, G.A. Sintomas de deficiências nutricionais em cupuaçuzeiro (Theobroma grandiflorum) cultivado em solução nutritiva. Scientia Agricola, v.5, p.407-414, 1994.

SCHWENDENER, C.M.; LEHMANN, J.; CAMARGO, P.B.; LUIZÃO, R.C.C.; FERNANDES, E.C.M. Nitrogen transfer between high- and low-quality leaves on a nutrient-poor Oxisol determined by ${ }^{15} \mathrm{~N}$ enrichment. Soil Biology and Biochemistry, v.37, p.787-794, 2005.

SILVA, M.J.G. Boletim climatológico de Rondônia, ano 1999. 2.ed. Porto Velho: Secretaria de Estado do Desenvolvimento Ambiental, 2000. 20p.

SILVA, M.A.C.; NATALE, W.; MALHEIROS, E.B.; PAVINATO, A. Estabelecimento e validaçáo de normas DRIS para a cultura do algodão no Centro-Oeste do Brasil. Acta Scientiarum Agronomy, v.31, p.93-99, 2009.

SMITHSON, P.C.; McINTYRE, B,D.; GOLD, C.S.; SSALI, H.; NIGHT, G.; OKECH, S. Potassium and magnesium fertilizers on banana in Uganda: yields, weevil damage, foliar nutrient status and DRIS analysis. Nutrient Cycling in Agroecosystems, v.69, p. 43-49, 2004.

SPSS. SPSS Graduate Pack. Version 16.0. Chicago: SPSS, 2009.

WADT, P.G.S. Análise foliar para recomendação de adubação em culturas agrícolas. In: PRADO; R.M.; ROZANE, D.E.; VALE, D.W.; CORREIA, M.A.R.; SOUZA, H.A. (Org.). Nutrição de plantas. Diagnose foliar em grandes culturas. Jaboticabal: Fundanesp, 2008. v.1, p.115-133.

WADT, P.G.S. Relationships between soil class and nutritional status of coffee crops. Revista Brasileira de Ciência do Solo, v.29, p.227-234, 2005.

WADT, P.G.S.; LEMOS, C.O. Medidas de acurácia para diagnósticos nutricionais e seu impacto no manejo das adubaçóes. In: PRADO, R.M.; CECÍlIO FILHO, A.B.; CORREIA, M.A.R.; PUGA, A.P. (Ed.). Nutrição de plantas: diagnose foliar em hortaliças. Jaboticabal: Universidade Estadual Paulista, 2010. p.213-236.

WADT, P.G.S.; SILVA, D.J. Acurácia do diagnóstico nutricional de pomares de mangueiras obtido por três fórmulas DRIS. Pesquisa Agropecuária Brasileira, v.45, p.1180-1188, 2010.

WADT, P.G.S.; SILVA, D.J.; MAIA, C.E. ; TOM JUNIOR, J.B.; PINTO, P.A.C.; MACHADO, P.L.O.A. Modelagem de funçôes no cálculo dos índices DRIS. Pesquisa Agropecuária Brasileira, v.42, p. 57-64, 2007. 